\title{
Development of an injectable PHBV microparticles-GG hydrogel hybrid system for regenerative medicine
}

\author{
Daniela P. Pacheco a,b,c,*, Maria H. Amaral ${ }^{c}$, Rui L. Reis ${ }^{\mathrm{a}, \mathrm{b}}$, Alexandra P. Marques ${ }^{\mathrm{a}, \mathrm{b}}$, \\ Vítor M. Correlo ${ }^{\mathrm{a}, \mathrm{b}, *}$ \\ a 3B's Research Group - Biomaterials, Biodegradables and Biomimetics, University of Minho, Headquarters of the European Institute of Excellence on Tissue \\ Engineering and Regenerative Medicine, AvePark, 4806-909 Taipas, Guimarães, Portugal \\ b ICVS/3B's - PT Government Associate Laboratory, Braga/Guimarães, Portugal \\ ${ }^{\mathrm{c}}$ Laboratory of Pharmaceutical Technology/Centre of Research in Pharmaceutical Sciences, Faculty of Pharmacy, University of Porto, 4050-313 Porto, Portugal
}

\section{A R T I C L E I N F O}

\section{Article history:}

Received 22 August 2014

Received in revised form 13 November 2014

Accepted 14 November 2014

Available online 18 November 2014

\section{Keywords:}

Polyhydroxybutyrate-co-hydroxyvalerate

Gellan gum

Microparticulate systems

Injectable hydrogel

Regenerative medicine

\begin{abstract}
A B S T R A C T
Uncontrollable displacements that greatly affect the concentration of active agents at the target tissues are among a major limitation of the use of microparticulate drug delivery systems (DDS). Under this context a biphasic injectable DDS combining poly(hydroxybutyrate-co-hydroxyvalerate) (PHBV) microparticles (MPs) and a gellan gum (GG) injectable hydrogel is herein proposed for the localized delivery and long-term retention of MPs carrying hydrophilic and hydrophobic model active agents. A double emulsion-solvent evaporation method was adopted to develop the PHBV MPs, carrying bovine serum albumin (BSA) or dexamethasone (Dex) as hydrophilic and hydrophobic active agents' models, respectively. Moreover, this method was modified, together with the properties of the hydrogel to tailor the delivery profile of the active agents. Variations of the composition of the organic phase during the process allowed tuning surface topography, particle size distribution and core porosity of the PHBV MPs and, thus, the in vitro release profile of Dex but not of BSA. Besides, after embedding hydrogels of higher GG concentration led to a slower and more sustained release of both active agents, independently of the processing conditions of the microparticulate system.
\end{abstract}

(c) 2014 Elsevier B.V. All rights reserved.

\section{Introduction}

The design and production of efficient drug delivery systems (DDS) are of vital importance in tissue engineering (TE) and regenerative medicine (RM) (Biondi et al., 2008). Ideally, DDS intend to sustain a particular active agents' concentration in patients' blood and/or tissues for a defined and extended time, avoiding repeated administration (Amass et al., 1998; Kost and Langer, 2001). Injectable DDS that have been proposed so far rely on liposomes (Monteiro et al., 2013), micelles (Xu et al., 2013), polymeric micro- and nanoparticles (Kozielski et al., 2013; Liang et al., 2013), cyclodextrins (Dhule et al., 2012), among others (Oliveira et al., 2011).

\footnotetext{
* Corresponding author at: 3B's Research Group - Biomaterials, Biodegradables and Biomimetics, Department of Polymer Engineering, University of Minho, Headquarters of the European Institute of Excellence on Tissue Engineering and Regenerative Medicine, AvePark, Taipas, 4806-909 Guimarães, Portugal; ICVS/3B's, Associate Laboratory, PT Government Associate Laboratory, Guimarães, Braga, Portugal. Tel.: +351 918236302.

E-mail address: vitorcorrelo@dep.uminho.pt (V.M. Correlo).
}

A number of different polymers, either synthetic or natural, have been exploited for the production of biodegradable DDS. These systems are cleaved through chemical or enzyme-catalyzed hydrolysis thus allowing the release of the active agent in a controlled manner and at a sustained concentration, within its therapeutic window. The release profiles of the active agents from biodegradable polymers, in addition to a dependence with the degradation mechanism and kinetics of the polymers (Ciçek et al., 1995; Mi et al., 2002; Zhang and Chu, 2002), can be controlled by a number of other factors, such as physicochemical properties of the polymers and active agents (Calandrelli et al., 2002; Abraham et al., 2003), thermodynamic compatibility between the polymers and active agents (Liu et al., 2004), as well as the shape of the devices (Chen and Lee, 2001; Tunón et al., 2003; Fulzele et al., 2004).

Among the biodegradable polymers that have been explored for the production of DDS, poly(lactide-co-glycolide) (Wen et al., 2013), poly(L-lactic acid) (Correia et al., 2013), chitosan (Champa and Bhat, 2010), alginate (Iwanaga et al., 2013), starch-poly- $\varepsilon$-caprolactone (Balmayor et al., 2009) and polyhydroxyalkanoates (PHAs) are the most used. From the PHA family, polyhydroxybutyrate (PHB) and its co-polymer with hydroxyvalerate (PHBV) in particular, in addition 
to TE applications (Zonari et al., 2014), have been also proposed as delivery systems for various active agents (Shishatskaya et al., 2008; Chen and Tong, 2012; Kabilan et al., 2012). PHAs microparticles (MPs) have been developed by different microencapsulation approaches that rely on solvent-evaporation methods, emulsification-diffusion, among others. The first approach offers advantages over other methods, since it is highly reproducible, presents the ability to serve as carriers of hydrophilic and hydrophobic active agents with high incorporation efficiency, which release profile can also be controlled by the processing factors (Ogawa et al., 1988; Yeo et al., 2001). On the other hand, the emulsification-diffusion is a very versatile technique, in which different types of emulsions can be used, though oil/water emulsions are of interest because they use water as the nonsolvent, simpler and more economic, since it facilitates the washing step and minimizes agglomeration (Pinto Reis et al., 2006). However, this method can only be applied to hydrophobic active agents, and limitations are imposed by the scale-up of the high energy requirements in homogenization (Soppimath et al., 2001). Relying on these microencapsulation methods, different applications have been proposed. In studies of antibiotic delivery, gentamicin was incorporated into PHAs MPs to prevent infections associated with bone implants and scaffolds (Francis et al., 2011), whilst RodríguezContreras produced PHAs MPs to delivery doxycycline (RodríguezContreras et al., 2013). Moreover, Poletto and co-workers adopted the emulsification-diffusion technique using a binary mixture of different proportions of chloroform and ethanol aiming to decrease the size of the particles (Poletto et al., 2008).

Although the microparticulate systems present many advantages, upon injection they are continuously exposed to external mechanical deformation, leading to uncontrollable displacements (Fitzgerald et al., 1987; Griffith, 2000; Chan and So, 2005). This greatly affects the concentration of active agents at the target tissues, which can lead to impaired outcomes, such as, for example, limited tissue regeneration (Lemperle et al., 2004). Moreover, when in free movement the particulate system can be expelled before the release of the active agent. Therefore, extensive efforts are being made to tackle this issue while harnessing the advantages of particulate systems, including their minimally invasive administration and the controlled delivery of the active agent. In this sense, the incorporation of MPs in injectable hydrogels was hypothesized in order to develop a minimallyinvasive DDS that would provide MPs localized delivery avoiding uncontrolled migration through the human body, along with a sustained release of an active agent.

From the clinical point of view, injectable hydrogels formed by in situ polymerization are highly desirable due to: (1) enabling minimally invasive application; (2) high capacity to adapt to the tissue defect; and (3) high compatibility with incorporated active agents and cells if desired (Jin, 2012). In this regard, gellan gum (GG) hydrogels that have been shown to be a suitable platform to support and deliver cells in different TE applications as extensively characterized in our Group, were selected (Coutinho et al., 2010; Oliveira et al., 2010; da Silva et al., 2014). The double emulsificationsolvent evaporation method described by Ogawa et al. (1988) was adopted to develop PHBV MPs, carrying bovine serum albumin (BSA) or dexamethasone (Dex) as hydrophilic and hydrophobic active agents' models, respectively. This method is highly reproducible and has been extensively used to create particles with a spherical and inner core-shell structure that can serve as carrier of water-soluble and insoluble active agents. The characteristics of the produced MPs are largely dependent on the type of polymer (hydrophobic/hydrophilic) and active agent used. Thus, this method was modified, together with the properties of the hydrogel to tailor the delivery profile of the active agents. In this way, a new in situ gelling injectable system, incorporating PHBV
MPs loaded with both relevant hydrophilic and hydrophobic molecules, which allows their localized and controlled release is herein proposed.

\section{Materials and methods}

\subsection{Preparation of $P H B V M P S$}

The PHBV (molecular weight $(\mathrm{Mw})=425,692 \mathrm{~g} \mathrm{~mol}^{-1}$ ) $(\mathrm{PHB}$ Industrial S.A., Brazil) MPs were prepared by using a modification of the double emulsification-solvent evaporation method previously described by Ogawa (Ogawa et al., 1988). Briefly, $3 \mathrm{~mL}$ of a $0.5 \%(\mathrm{w} / \mathrm{v})$ poly(vinyl alcohol) (PVA) (Sigma-Aldrich, Germany) aqueous solution (W1) was mixed with $15 \mathrm{~mL}$ of a $3 \%(\mathrm{w} / \mathrm{v})$ PHBV solution in chloroform-ethanol (O). Two different ratios of chloroform-ethanol were used in order to verify its effect over the release profile of the active agents, namely 100:0 chloroform: ethanol (absence of ethanol) and 90:10 chloroform:ethanol (presence of ethanol). The mixture was emulsified for 3 min using an Ultra-Turrax T18 (IKA-Werke, Germany) in order to obtain the first emulsion (W1/O). The primary emulsion was then dropped into $150 \mathrm{~mL}$ of $1 \%(\mathrm{w} / \mathrm{v}$ ) PVA solution (W2) and homogenized (RW 16 basic IKA-Werke, Germany) during 5 min forming the secondary emulsion (W1/O/W2). The final W1/O/W2 emulsion was left to evaporate under magnetic stirring and the obtained MPs were collected by centrifugation, washed at least three times with deionized water and freeze-dried. BSA was dissolved at a concentration of $0.1 \%(\mathrm{w} / \mathrm{v})$ within the first aqueous phase (W1), whilst Dex was dissolved in the organic phase at a final concentration of $3.3 \times 10^{-3} \%(\mathrm{w} / \mathrm{v})$. Unloaded-MPs were also prepared, as control, using the same protocol previously described, but in the absence of the active agents.

\subsection{Incorporation of PHBV MPs in GG hydrogels}

For the preparation of the injectable GG hydrogels, a previously described procedure was followed (da Silva et al., 2014). Gelzan CM (Sigma-Aldrich, Germany) powder was mixed at room temperature with deionized water at two different concentrations, $0.75 \%$ $(\mathrm{w} / \mathrm{v})$ and $1.25 \%(\mathrm{w} / \mathrm{v})$, under constant stirring. The solution was heated at $90^{\circ} \mathrm{C}$ and kept at this temperature for $30 \mathrm{~min}$. Afterwards, $30 \mathrm{mg}$ of active agent-loaded PHBV MPs were dispersed with $20 \mathrm{~mL}$ of GG solution. Subsequently, calcium chloride (VWR, USA) was added to this solution at a concentration of $0.18 \%(w / v)$ to act as crosslinker. Finally, the solution was left at room temperature during approximately one hour, allowing the formation of the gel, which was then maintained in a phosphatebuffered saline (PBS) solution.

\subsection{Quantification of the MPs' production yield}

The yield of the particles prepared under the different conditions defined in Section 2.1 was calculated using Eq. (1).

$\operatorname{yield}(\%)=\frac{w_{x}}{w_{y}} \times 100$

The yield was calculated based on the weight of MPs $\left(w_{x}\right)$ and the weight of the compounds used to prepare the $\operatorname{MPs}\left(w_{y}\right)$, polymer or polymer plus active agent.

\subsection{Quantification of the incorporation efficiency of Dex and BSA into the PHBV MPS}

Aiming at determining the BSA incorporation efficiency (IE) into the MPs, $10 \mathrm{mg}$ of BSA- or Dex-loaded MPs were completely 
dissolved in chloroform with vigorous shaking and at room temperature for $24 \mathrm{~h}$. This allows the dissolution of PHBV and at the same time permits the release of the entrapped active agent. Then, $10 \mathrm{~mL}$ of PBS was added in order to dissolve the BSA/Dex. After centrifugation, the supernatant was collected and filtered through a $0.2 \mathrm{~mm}$ membrane. The concentration of the incorporated BSA was measured using the Micro BCA ${ }^{\mathrm{TM}}$ Protein Assay Kit (Pierce, IL) following manufacturer's instructions. Optical density of the BSA- and Dex-containing solutions was measured at 562 and $242 \mathrm{~nm}$, respectively, in a Synergy HT multi-detection microplate reader (Bio-Tek's Gen5 ${ }^{\mathrm{TM}}$, USA). The amounts of BSA and Dex within the MPs were obtained from the calibration curves. The IE of the active agents within PHBV MPs was calculated using Eq. (2) as follows:

$\operatorname{IE}(\%)=\frac{[\text { BSA or Dex }] \text { within MPs }}{\text { Initial }[\text { BSA or Dex }]} \times 100$

\subsection{In vitro release studies}

In vitro release of BSA and Dex from PHBV MPs was evaluated up to 21 days. For that, $30 \mathrm{mg}$ of MPs were incubated in $10 \mathrm{~mL}$ of PBS $(0.01 \mathrm{M}, \mathrm{pH} 7.4)$, and maintained in a precision water bath (Grant, UK) at $60 \mathrm{rpm}$ and $37.0 \pm 0.5^{\circ} \mathrm{C}$. At defined time points, $1 \mathrm{~mL}$ of supernatant was collected and an equivalent amount of fresh PBS at $37.0 \pm 0.5^{\circ} \mathrm{C}$ was added to maintain the total volume of the sample. The amount of released BSA and Dex was determined as described above. A similar procedure was followed to determine the release profile of BSA and Dex from the PHBV MPs embedded within the GG hydrogels.

\subsection{Analysis of the kinetics and mechanism of release}

The release mechanism of BSA and Dex from both PHBV MPs and PHBV MPs embedded in GG hydrogels was analyzed by applying the results in different kinetic models (Costa and Sousa Lobo, 2001). The zero-order that explains the active agent release rate as an independent phenomenon of the active agent concentration (Donbrow and Samuelov, 1980). The first-order usually applied to active agent dissolution studies (Gibaldi and Feldman, 1967; Wagner, 1969). The Higuchi that is concerned on the release of water soluble and low soluble active agents incorporated in semi-solid and/or solid matrices, and describes the active agent release as a diffusion process based on Fickian diffusion (Higuchi, 1961, 1963). And the Korsmeyer-Peppas, generally used to analyze the release of pharmaceutical polymeric dosage forms, when the release mechanism is not well known or when more than one type of release phenomena could be involved (Korsmeyer and Peppas, 1981; Korsmeyer et al., 1983; Costa and Sousa Lobo, 2001), were the applied models. The determination coefficient $\left(R^{2}\right)$ was used as an indicator of the best fitting of the data for each model. $R^{2}$ and model parameters of the release profiles were calculated using SigmaPlot 12.5 software (Systat Software Inc., USA).

\subsection{Laser diffraction spectrometry}

The size distribution of the prepared MPs was measured by laser diffraction spectrometry (Coulter LS 230, Coulter Electronics, USA). The dried samples were suspended in a $0.2 \%(\mathrm{w} / \mathrm{v})$ Tween ${ }^{\circledR}$ 80 (Sigma-Aldrich, Germany) solution and sonicated for $5 \mathrm{~min}$ with an ultra-sound probe (Sonoswiss SW 6H, Sonoswiss ${ }^{\circledR}$ AG,

\begin{tabular}{lcc}
\hline $\begin{array}{l}\text { Processing Conditions } \\
\text { Chloroform:Ethanol ratio }\end{array}$ & $\begin{array}{c}\text { Yield, \% } \\
(\text { mean } \pm \text { SD) }\end{array}$ & $\begin{array}{c}\text { Particle Size Distribution, } \boldsymbol{\mu m} \\
\text { (mean } \pm \text { SD) }\end{array}$ \\
\hline PHBV (100:0) & $95.7 \pm 2.32^{*}$ & $41.9 \pm 20.2^{* *}$ \\
PHBV (90:10) & $90.3 \pm 2.43^{*}$ & $57.6 \pm 29.1^{* *}$ \\
\hline
\end{tabular}
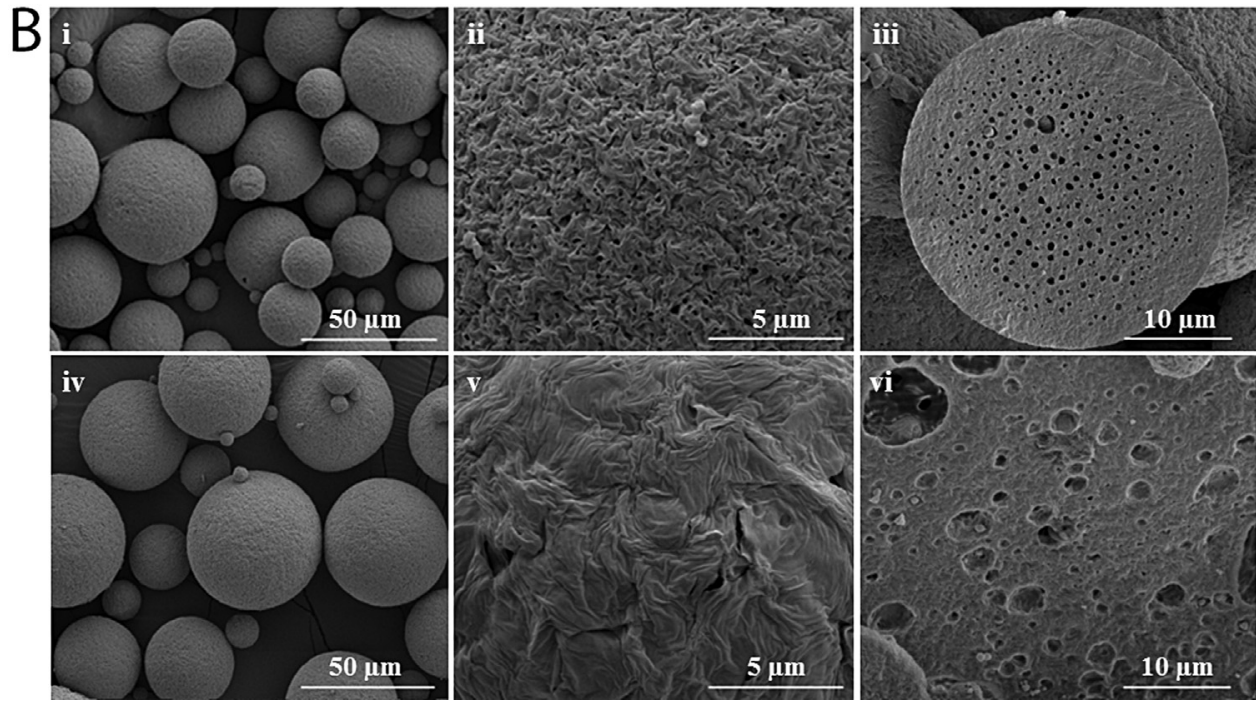

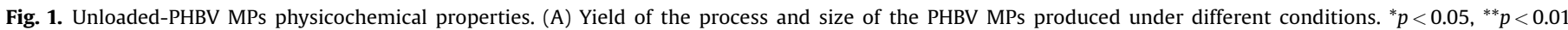

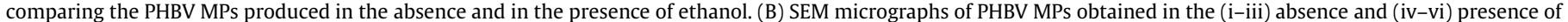

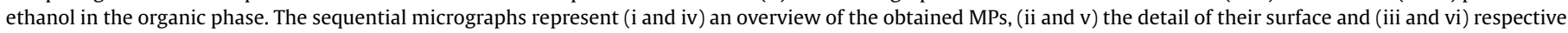
internal morphology. 
Switzerland) before measurement. The obtained homogeneous suspension was used for the particle size distribution analysis.

\subsection{Fourier transform infrared spectroscopy}

Fourier transform infrared spectroscopy (FTIR) analyses, under Transmission mode, were performed in order to analyze PHBV MPs (unloaded, loaded and after release) chemical composition. With this purpose, $1 \mathrm{mg}$ of MPs were mixed with $40 \mathrm{mg}$ of potassium bromide $(\mathrm{KBr})$ and then processed into a disc in a manual press (161-1100 hand press, Pike technologies, Madison, WI). FTIR-KBr spectra (IR Prestige 21, Shimadzu, Japan) were recorded at 40 scans with a resolution of $4 \mathrm{~cm}^{-1}$, from 1400 to $4000 \mathrm{~cm}^{-1}$.

\subsection{Scanning electron microscopy}

The morphology of the PHBV MPs and PHBV MPs embedded within GG hydrogels was analyzed before and after the in vitro release studies, by scanning electron microscopy (SEM) using a S360 microscope (Leica Cambridge, UK). Before being analyzed, the different structures were gold sputter coated (Fisons instruments, UK) for $2 \mathrm{~min}$ at $15 \mathrm{~mA}$. Micrographs were recorded at $5.0 \mathrm{kV}$ and different magnifications.

\subsection{Rheological analysis}

Cone-plate rheometry was conducted for GG hydrogels with and without embedded PHBV MPs to assess their rheological behavior as function of temperature and time. For this purpose, in each measurement, a volume of $2 \mathrm{~mL}$ of the sample was placed in the bottom plate of the rheometer (Malvern Kinexus Rotational Rheometer, UK) and allowed to stabilize for $1 \mathrm{~min}$ at a constant temperature of $5{ }^{\circ} \mathrm{C}$ before starting the analysis. Measurements were performed by increasing the temperature from 5 to $50{ }^{\circ} \mathrm{C}$ (at a heating rate of $1^{\circ} \mathrm{C} / \mathrm{min}$ ) and applying a constant shear stress of $0.1 \mathrm{~Pa}$.

\subsection{Statistical analysis}

The results of at least three independent experiments are presented as mean \pm standard deviation (SD). The yield, particle size and IE data were analyzed using the one-way analysis of variance (ANOVA) $(\alpha=0.05)$ and Tukey's multiple comparison test using Graphpad Prism version 5 (Graphpad Software, USA). Significance differences were set for $p<0.05$.

\section{Results}

\subsection{Effect of processing method over PHBV MPs properties}

The PHBV MPs were produced following a double emulsification-solvent evaporation method using a mixture of chloroform: ethanol in two different proportions in order to assess the effect of the processing conditions over the MPs properties and subsequent incorporation capacity and release profile of BSA and Dex. The yield in the preparation of PHBV MPs (Fig. 1A) revealed to be significantly $(p<0.05)$ affected by the solvent composition. The results showed that the presence of ethanol in the organic phase led to a decreased of $6 \%$ in the yield. Likewise, the size distribution of the MPs was also significantly $(p<0.01)$ affected by the processing conditions. The mean particle size of unloaded-MPs obtained in the presence of ethanol (around $58 \mu \mathrm{m}$ ) was found to be larger than in its absence (around $42 \mu \mathrm{m}$ ), with a distribution of range from 15.6 to $76.4 \mu \mathrm{m}$ and 5.6 to $91.1 \mu \mathrm{m}$, respectively.

Concerning the morphology of the produced MPs (Fig. 1B), perfect spherical shape MPs were obtained independently of the processing conditions (Fig. 1B; i and iv). However, a detailed analysis of MPs surface topography revealed rough surfaces, both

\begin{tabular}{cccccc}
\hline $\begin{array}{l}\text { Processing Conditions } \\
\text { Chloroform:Ethanol ratio }\end{array}$ & \multicolumn{2}{c}{$\begin{array}{c}\text { Yield, \% } \\
\text { (mean } \pm \text { SD) }\end{array}$} & \multicolumn{2}{c}{$\begin{array}{c}\text { Particle Size Distribution, } \boldsymbol{\mu m} \\
\text { (mean } \pm \text { SD) }\end{array}$} \\
\hline Entrapped active agent & BSA & Dex & BSA & Dex \\
PHBV (100:0) & $88.8 \pm 5.50$ & $95.4 \pm 1.36$ & $78.5 \pm 78.5^{* * *}$ & $55.0 \pm 39.4^{*}$ \\
PHBV (90:10) & $79.0 \pm 9.83$ & $89.9 \pm 5.64$ & $63.0 \pm 42.2$ & $58.3 \pm 27.5$ \\
\hline
\end{tabular}

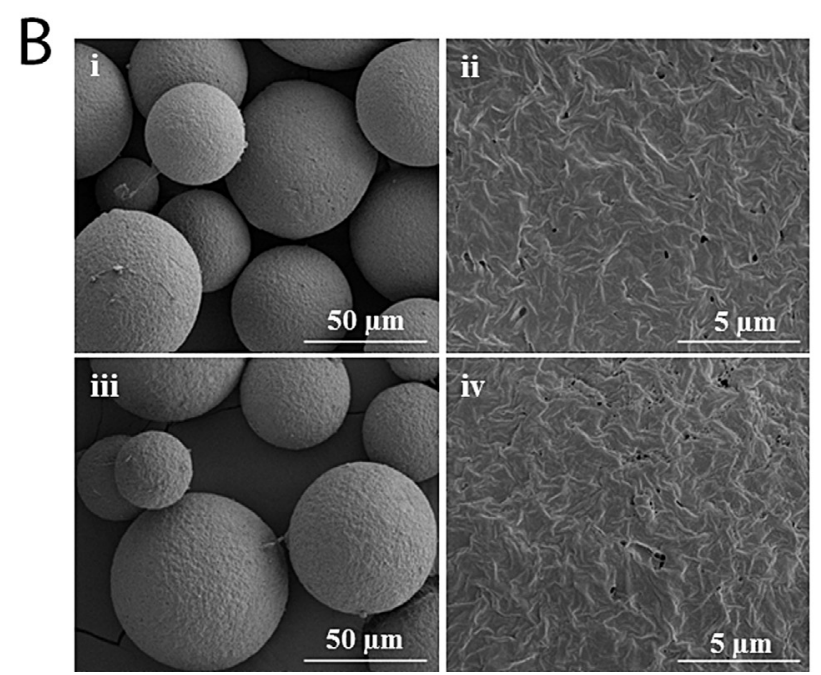

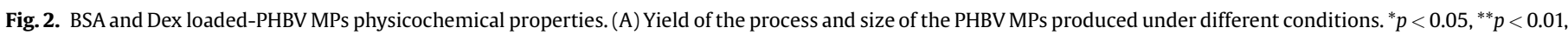

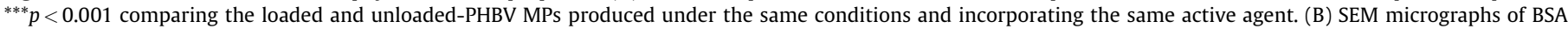

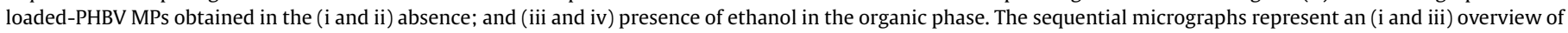
the obtained MPs and (ii and iv) the detail of their surface. 
with the presence of some micro-size pores, but with different topography (Fig. 1B; ii and v). Moreover, both in the presence and absence of ethanol, MPs presented a porous core (Fig. 1B; iii and vi). Nonetheless, the addition of ethanol to the organic phase led to the formation of larger pore in the inner structure (Fig. 1B; vi).

\subsection{Loaded-MPs properties}

\subsubsection{BSA and Dex entrapment}

The impact of the incorporation of active agents within PHBV MPs over the process yield and particle size was determined (Fig. 2A). While no significant differences were observed in terms of yield when producing unloaded-PHBV MPs (Fig. 1A) and active agent-loaded MPs (Fig. 2A) ( $p>0.05)$, the size of the loaded-PHBV MPs was affected by the incorporation of the active agents (Fig. 2A). In the absence of ethanol, the mean size of the loadedPHBV MPs, independently of the incorporated agent, was significantly higher than the unloaded-MPs. This difference was not observed in the presence of ethanol. Furthermore, the mean MPs size significantly increased with the molecular weight (Mw) of the active agent that was incorporated. Thus, the BSA-loaded PHBV MPs exhibited a higher mean particle size when compared with the BSA-loaded PHBV MPs (Fig. 2A).

The SEM analysis of the loaded-PHBV MPs confirmed not only the particle sizemeasurements, butalso the maintenance of the spherical morphology after incorporation of the active agents (Fig. 2B; i and iii), which was independent of the incorporated molecule.

The IE of BSA and Dex within the PHBV MPs obtained under different experimental conditions revealed to be independent of the processing conditions $(p>0.05)$. An IE around 66\% was achieved for BSA both in presence and absence of ethanol (Fig. 3A). Although not significantly different $(p>0.05)$, the presence of ethanol in the organic phase resulted in higher (around 92\% vs 82\%) IE of Dex. Moreover, the Dex IE was significantly higher than BSA.

The entrapment of BSA and Dex within PHBV MPs was further confirmed by FTIR analysis (Fig. 3B and C). The IR peaks of pure BSA at 1652 and $1531 \mathrm{~cm}^{-1}$ are assigned to stretching vibration of amide I (mainly $\mathrm{C}=\mathrm{O}$ stretching vibrations), and amide II (the coupling of bending vibrate of $\mathrm{N}-\mathrm{H}$ and stretching vibrate of $\mathrm{C}-\mathrm{N}$ ) bands, respectively. The FTIR spectra of BSA-loaded PHBV MPs revealed these characteristic bands of both the polymer and the protein, indicating that the hydrophilic active agent was successfully incorporated. Similarly, the FTIR analysis also confirmed the successful entrapment of Dex inside the PHBV MPs (Fig. 3C), since its characteristic peaks are visible at 1700 and $1660 \mathrm{~cm}^{-1}$, which corresponds to the carbonyl stretching vibration of C-17 dihydroxiacetone side chain, and a peak at $1660 \mathrm{~cm}^{-1}$ due to carbonyl stretching vibration of C3 A-ring associated with the $1630 \mathrm{~cm}^{-1} \mathrm{O}-\mathrm{H}$ stretching band of interlamellar water.

\subsubsection{BSA release from $P H B V M P S$}

The release profile from BSA-loaded PHBV MPs produced in the absence of ethanol indicated that $76 \%$ of the BSA was released within the first $8 \mathrm{~h}$ reaching a release of approximately $78 \%$ of the incorporated BSA that was sustained up to day 21 (Fig. 4A). Similarly, the BSA loaded-MPs produced in the presence of ethanol released about $78 \%$ of the incorporated BSA within the first $8 \mathrm{~h}$. After 21 days of incubation, approximately $80 \%$ of BSA was released from BSA-loaded PHBV MPs. These results fit the Higuchi model. The BSA release was confirmed by FTIR analysis (Fig. 4B). The spectra of BSA-loaded PHBV MPs produced both in the absence and presence of ethanol, revealed a reduction in the intensity of the BSA characteristic bands (Fig. 4B) up to 21 days. At this time point, the presence these were still verified corresponding to the remaining protein entrapped in the MPs.

The MPs incorporating BSA, monitored by SEM, showed minor morphological and topographical alterations after the in vitro release studies (Fig. 5C). Moreover, MPs structure and integrity was maintained.

\subsubsection{Dex release from $P H B V M P s$}

The release profile of Dex from Dex-loaded PHBV MPs produced in the absence of ethanol indicated that $55 \%$ of Dex was released within the first $8 \mathrm{~h}$ and a maximum of approximately $69 \%$ of the incorporated Dex at released up to day 21. In opposition, $89 \%$ of the incorporated Dex in the MPs produced in the presence of ethanol was released within the first $8 \mathrm{~h}$ and no Dex was present in the MPs after 5 days. The release of Dex from the particles obtained in the absence of ethanol followed the Higuchi model, whereas the

\begin{tabular}{llcc} 
A & $\begin{array}{l}\text { Processing Conditions } \\
\text { Chloroform:Ethanol ratio }\end{array}$ & \multicolumn{2}{c}{$\begin{array}{c}\text { Incorporation Efficiency, \% } \\
(\text { mean } \pm \text { SD) }\end{array}$} \\
\cline { 2 - 4 } & Entrapped active agent & BSA & Dex \\
\hline PHBV (100:0) & $66.2 \pm 3.33$ & $81.6 \pm 8.41$ \\
PHBV (90:10) & $65.5 \pm 1.72$ & $92.3 \pm 2.17$ \\
\hline
\end{tabular}
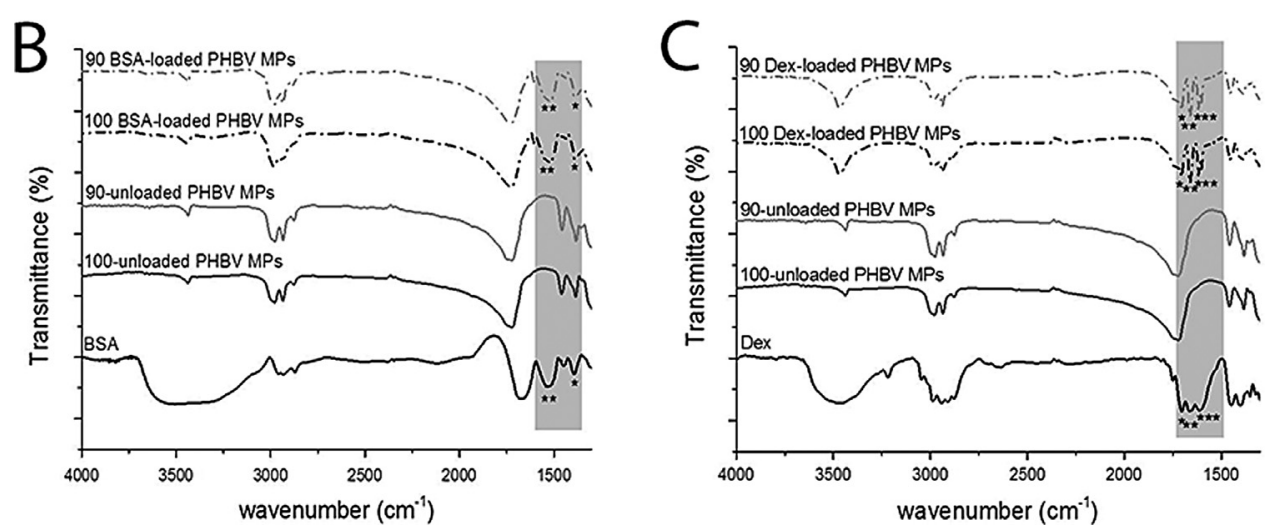

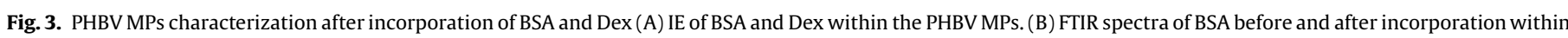

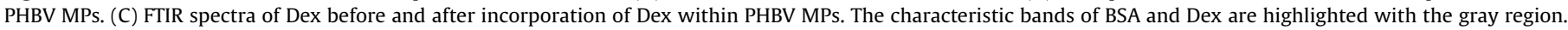



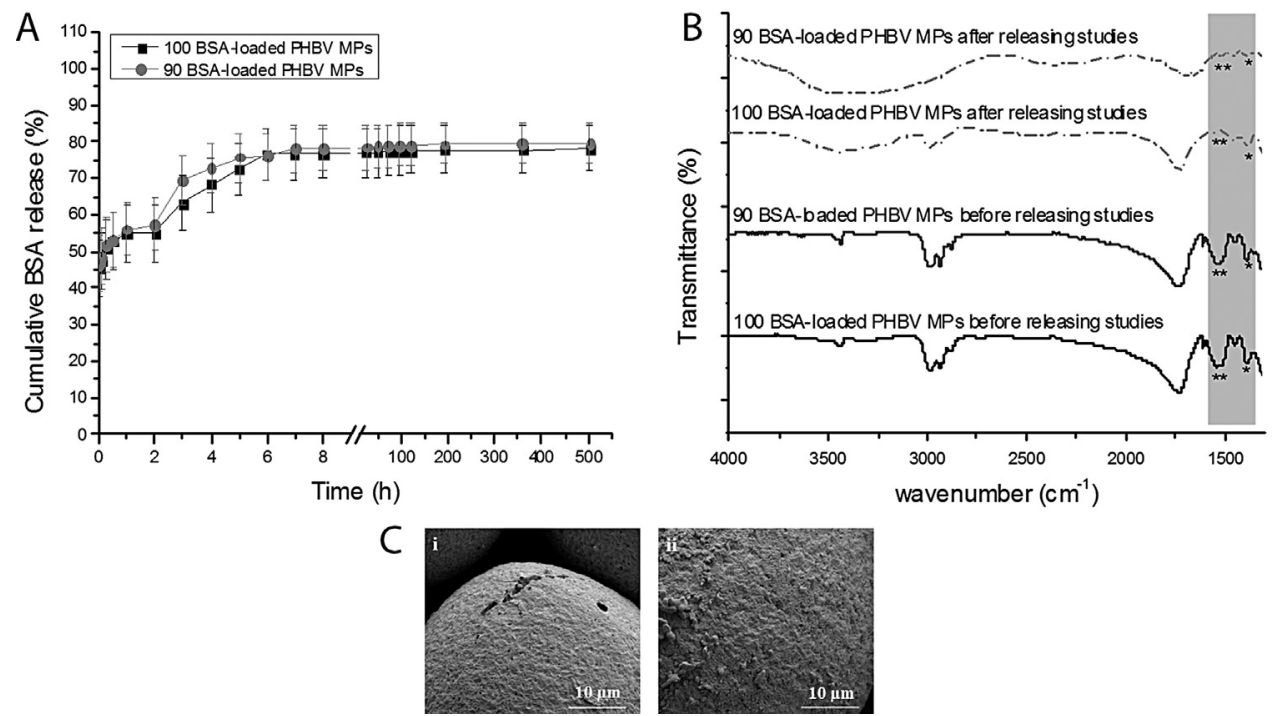

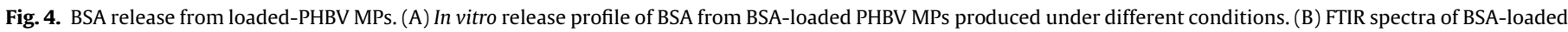

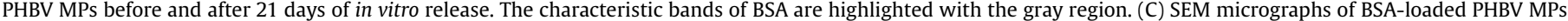
obtained in the (i) absence and (ii) presence of ethanol, after 21 days of in vitro release studies.
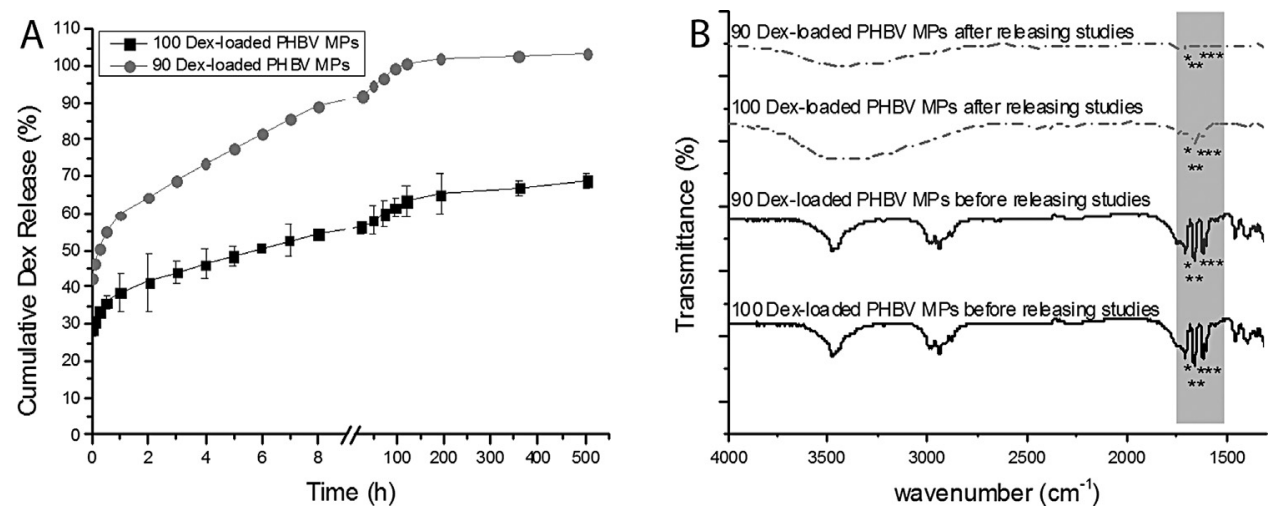

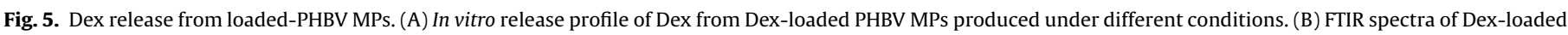
PHBV MPs before and after 21 days of in vitro release. The characteristic bands of Dex are highlighted with the gray region.

release kinetics of Dex from the MPs produced in the presence of ethanol fitted the Korsmeyer-Peppas model. Therefore, in this case, since $n$ is smaller than 0.43 , a classical Fickian diffusion is predicted.

The Dex release from the PHBV MPs was confirmed by FTIR analysis (Fig. 5B). While the FTIR spectra of Dex-loaded PHBV MPs, produced in the absence of ethanol, revealed a reduction in the intensity of the Dex characteristic bands due to the release but a still present at day 21 , those bands were absent in the spectra corresponding to the Dex-loaded PHBV MPs produced in the presence of ethanol after the release (Fig. 5B).

As observed for the MPs incorporating BSA, the Dex-loaded PHBV MPs morphology and shape were not affected by the Dex release (data not shown).
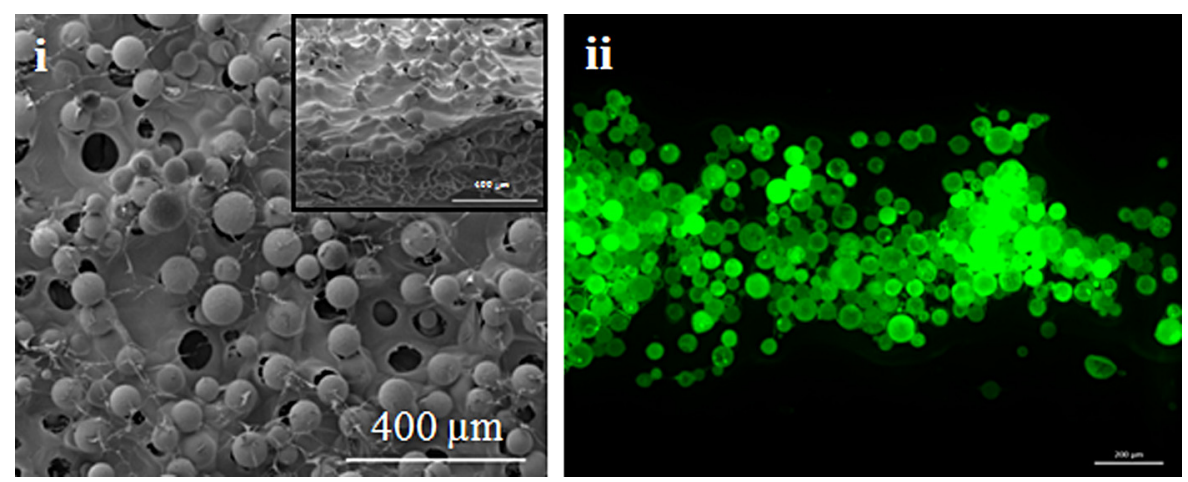

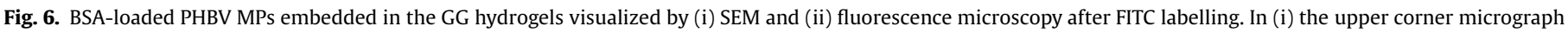
represents a cross-section of the biphasic structure. 


\subsection{Injectable GG/PHBV MPS}

\subsubsection{Injectable system properties}

The embedding of the PHBV MPs produced under different conditions in the $0.75 \%$ and $1.25 \%$ (w/v) GG solutions was confirmed. After gelation, the MPs were homogeneously dispersed within the hydrogel, maintaining their spherical shape (Fig. 6). Moreover, PHBV MPs were completely embedded within the GG matrices, as it is possible to observe in Fig. 6; ii.

The rheological analysis of the GG hydrogel with or without PHBV MPs, showed that, independently of the concentration of GG, the sol-gel transition temperature of the systems was around $39^{\circ} \mathrm{C}$ (Fig. 7) and gels were formed within $20 \mathrm{~s}$. This temperature was barely affected by the presence of the PHBV MPs within the hydrogel, however, as expected the viscosity of the $0.75 \%$ GG (Fig. 7A) was lower than the 1.25\% GG hydrogels (Fig. 7B). Moreover, it was observed that independently of the processing conditions, the presence of the microparticulate system within the hydrogel increased its viscosity, nevertheless, was not significantly.

\subsubsection{BSA release from the injectable GG/PHBV MPs system}

The impact of the incorporation of the MPs in the hydrogel over BSA release profile, showed that the presence of the matrix surrounding the MPs (Fig. 8A) almost eliminated the release burst observed for the single MPs suspensions (Fig. 4A). This effect was more notorious for the $1.25 \%(\mathrm{w} / \mathrm{v})$ of $\mathrm{GG} ; 35 \%$ of the BSA incorporated in the MPs produced in the absence of ethanol was released within the first $8 \mathrm{~h}$. In contrast, about $40 \%$ of the incorporated BSA was released from the loaded-MPs produced in the presence of ethanol within the first $8 \mathrm{~h}$. Both those values were the maximum released after 21 days. In contrast, the release
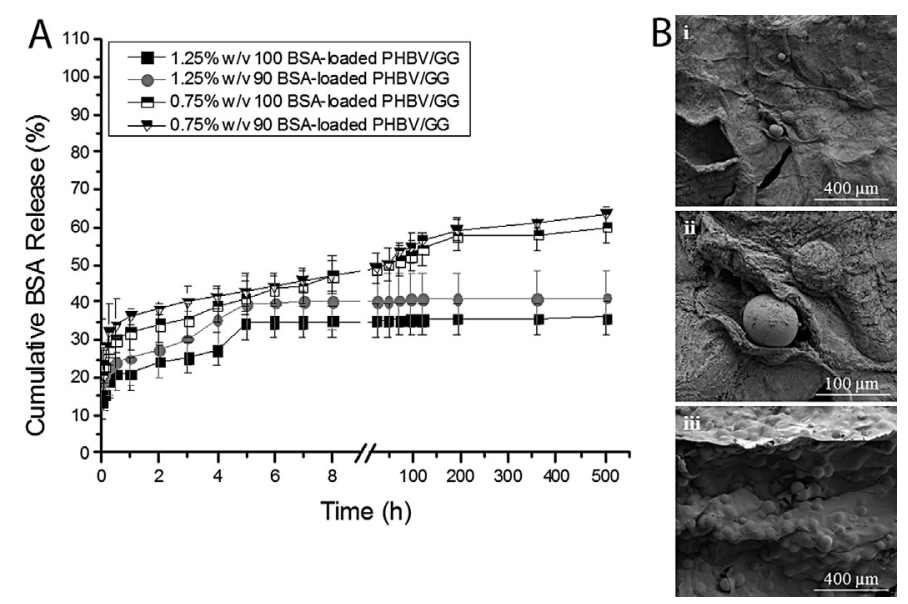

Fig. 8. (A) BSA release from loaded-PHBV MPs produced under different conditions and embedded in $0.75 \%$ and $1.25 \%$ GG hydrogels. (B) SEM micrographs of the GG hydrogel containing the BSA loaded-MPs after 21 days of in vitro release studies. The sequential images represent (i) an overview of the distribution of the particles within the hydrogel, (ii) a close up on the surface, and (iii) a cross-section.

profile of BSA from the MPs embedded in the $0.75 \%$ GG hydrogel indicated that $47 \%$ of protein was released within the first $8 \mathrm{~h}$ reaching a release of approximately $60 \%$ and $63 \%$ of the incorporated BSA at day 21, respectively for the BSA loaded-MPs produced in the absence and in the presence of ethanol. Interestingly, independently of the concentration of the GG hydrogel, the release kinetics suggested that the release of BSA from the tested formulations followed a zero-order release.
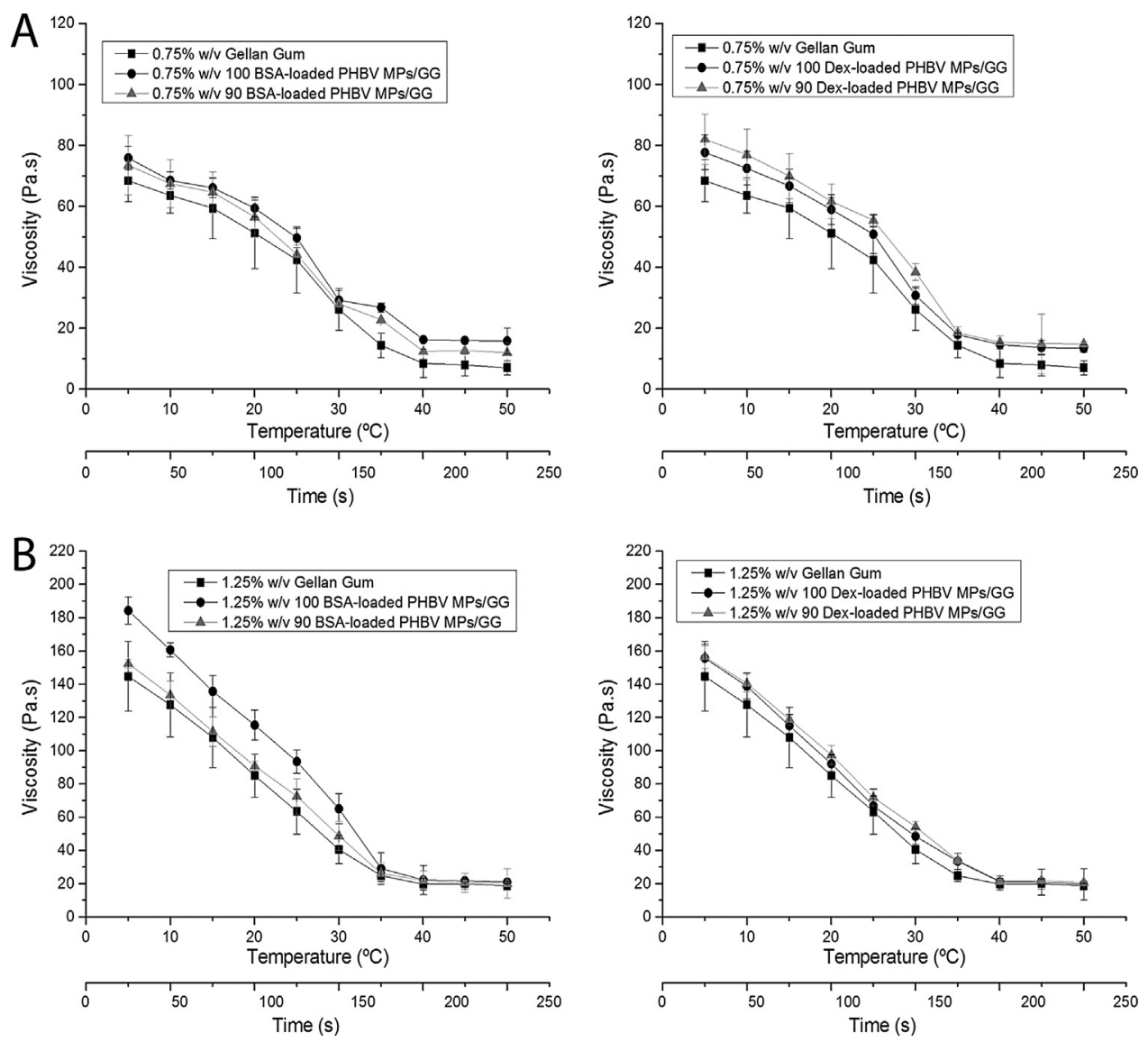

Fig. 7. Rheological behavior of (A) $0.75 \%$ and (B) $1.25 \%$ GG hydrogels, containing or not PHBV MPs produced under different conditions. 
After 21 days of immersion in PBS the system remained stable. The PHBV MPs were still embedded in the system (Fig. 8C) although the hydrogel revealed some signs of degradation. The surface erosion was apparent resulting in the formation of some cavities. No particles migration was observed after the 21 days of release studies.

\subsubsection{Dex release from the injectable GG/PHBV MPs system}

In accordance to what was observed for the BSA-loaded PHBV MPs embedded in the GG hydrogels, the Dex release profile (Fig. 9) confirmed that the GG hydrogel surrounding the Dex-loaded MPs eliminated the initial burst observed for the single MPs suspensions (Fig. 5A). Again, a higher effect observed for the $1.25 \%$ GG systems but in this case, the release profile was not dependent on the MPs processing conditions as no differences were observed between the systems produced in the presence and in the absence of ethanol. Both systems attained a release of around $26 \%$ of the incorporated Dex in the end of the 21 days. Regarding the release profile of Dex from $0.75 \%$ (w/v) GG hydrogels, dependence with the processing conditions was observed. The MPs produced in the absence of ethanol released around 20\% of the incorporated Dex within the first $8 \mathrm{~h}$ reaching approximately $43 \%$ of at day 21 . Similarly, the Dex loaded-MPs produced in the presence of ethanol released about $28 \%$ of the incorporated Dex within the first $8 \mathrm{~h}$. However, after 21 days of incubation approximately 52\% of Dex was released from this system. The release kinetics of all these formulations followed a Korsmeyer-Peppas model, suggesting a classical Fickian active agent diffusion since $n<0.43$.

Moreover, in similarity to what was observed during BSA release from GG hydrogels, no particle migration from the hydrogel was noticed.

\section{Discussion}

Aiming at developing a minimally-invasive DDS we designed a biphasic injectable DDS that can provide a localized delivery of MPs avoiding their uncontrolled migration through the human body, along with a prolonged release of an active agent. For this purpose, BSA- and Dex-loaded PHBV MPs, processed by a double emulsification-solvent evaporation method with modifications, were incorporated into injectable GG hydrogels with different concentrations.

The solvent type, the concentration of the emulsifier, the active agent/polymer ratio and the stirring rate are parameters known to affect the physicochemical properties of the MPs (Bazzo

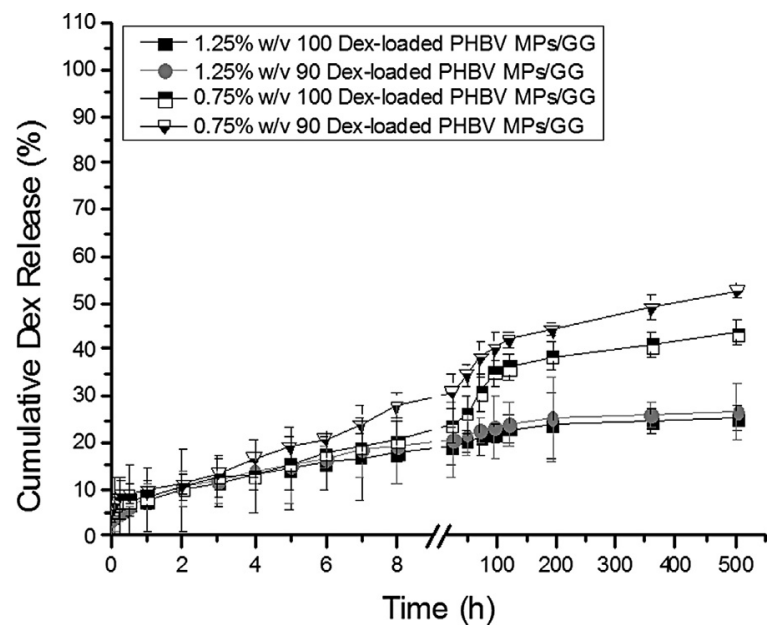

Fig. 9. Dex release from loaded-PHBV MPs produced under different conditions and embedded in $0.75 \%$ and $1.25 \%$ GG hydrogels. et al., 2008). In this regard, a mixture of different solvents, as organic phase, is herein proposed aiming to tailor the release profile of hydrophilic and hydrophobic agents by changing the properties of the particles. While chloroform was selected as the solvent for the PHBV, ethanol was aimed to improve the mutual solubility of the organic and the aqueous phases, based on the type of molecular interactions between the organic solvents (dipole-dipole) and between ethanol and water (hydrogen bond) (Poletto et al., 2008). The obtained PHBV MPs had sizes that ranged from 41.9 to $78.5 \mu \mathrm{m}$, depending on the presence of ethanol, as well as on the type of molecule incorporated. The size of the MPs in the presence of ethanol in the organic phase was higher than in its absence. These results are not in accordance to what was previously reported by Poletto et al. (2008) that showed a decrease in the size of PHBV nanoparticles when ethanol was used as a surface agent. The distinct results are probably due to the differences between the methods used to produce the particles; while they followed an emulsification-diffusion method, the double emulsification-solvent evaporation method herein proposed involves less high-speed homogenization, which was previously proved to have a tremendous effect on the particle size (Mukherjee et al., 2008). Furthermore, the concentration of PHBV used Poletto et al. (2008) was much smaller than the one we used. Higher polymer concentrations in the organic phase lead to increased viscosity of the solution and consequently lower dispersion thus larger MPs are produced (Kassab et al., 1997; Yang et al., 2001).

In addition to an effect over particle size, the solvents used during processing also impact the morphology and topography of the particles. It was previously reported that when the organic phase has increased miscibility with water, the interaction between emulsifier and the aqueous phase increases, thus particles with rougher surfaces are obtained (Sahoo et al., 2002). The roughness of the MPs surface has been also associated to the high crystallinity and fast precipitation of PHBV, after solvent evaporation from the internal phase of the emulsion (Bidone et al., 2009). The produced PHBV MPs showed a welldefined spherical shape independent of the processing conditions. However, the topography of the surface of the MPs was influenced by the presence of ethanol in the process. This finding is in accordance with what was stated by others that showed the ethanol improves the miscibility of both phases and therefore a better interaction with the emulsifier (Sahoo et al., 2002). The presence of ethanol also influenced the inner core porosity of the particles contrarily to the micro-size porosity found on the PHBV MPs surfaces observed for all the preparation conditions. The presence of ethanol on the organic phase led to larger core pores, which could be attributed to the hydrophobicity of PHBV. During the first emulsification, water droplets (first aqueous phase) interact with the ethanol present in the organic phase, but the same is not observed when the organic phase is composed only by chloroform. In this sense, the presence of ethanol in the organic phase leads to the formation of larger droplets inside the dispersed phase and consequently to larger pores after removal of the water droplets by freeze-drying, in accordance to what was previously reported in the literature (Zhu et al., 2007).

The incorporation of BSA and Dex within the PHBV MPs also affected their properties. An increase in the mean size was observed for the loaded-PHBV MPs, proportional to the MW of the incorporated active agent. The BSA-loaded MPs, incorporating the protein with a MW of $66 \mathrm{kDa}$, exhibited larger mean size than Dexloaded MPs that have entrapped a molecule with a MW of 392.46 Da.

The incorporation of active agents is highly dependent on the chemical structure and MW of the molecules, as well as on the hydrophobicity of the polymers (Park et al., 2005). In this study, BSA IE revealed to be independent of the organic phase 
composition, which was also observed for the IE of Dex. However, the active agent IE was affected by its MW, since Dex had a significantly higher IE than BSA. These results are in accordance with Lionzo et al. (Lionzo et al., 2007) that proposed a general single emulsification-solvent evaporation method for the incorporation of Dex within PHBV MPs.

The in vitro release profile of a molecule entrapped in polymeric MPs/matrix is controlled by a variety of factors, such as the solubility of the molecules in the surrounding media, its MW and mobility within the swollen polymeric network, as well as the interactions of the polymer with the active agent (Embleton and Tighe, 1993; Sah et al., 1994; Giunchedi et al., 1994; Igartua et al., 1997; Yang et al., 2001). Moreover, other features such as the morphology, size and size distribution of the particles also influence the release kinetics. These represent the ones that can be controlled by varying the processing conditions. Although PHAs have been described as high crystalline polymers, the reason for its slow degradation, excessive release rates from PHAs MPs have been previously and consistently reported, and assumed to be mainly dependent on active agent dissolution rather than matrix degradation (Bidone et al., 2009). This is in agreement with our results that confirmed structurally intact and spherical shape MPs after the release studies. We also found that the Dex amount released from the Dex-loaded PHBV MPs produced in the absence of ethanol was minor than for the MPs produced in the presence of ethanol. This finding was expected since the addition of ethanol to the organic phase led to the formation of particles with larger inner pores that facilitated the water penetration. Regarding the BSA, the release studies revealed no significant differences when ethanol was added to the organic phase, and a release of around $78 \%$ of the incorporated protein was observed after 21 days of immersion. Thus, it was also observed that the MW of the incorporated active agents did not only affect the IE but also the amount of active agent released. Due to its lower MW, Dex had higher ability to diffuse across the polymeric matrix, corroborating the fact that the amount of small molecules released is commonly higher than proteins.

In this sense, MPs composed by PHBV with tuned surface topography, size distribution and core porosity were obtained using simple variations of the composition of the organic phase during processing. The in vitro release profile of Dex but not of BSA, was also affected allowing the control over the amount of Dex release along the time.

Nonetheless, a biphasic systems with a uniform distribution of PHBV MPs across the GG matrix, and the ability to be injected into the organism and with gelation occurring approximately at the body temperature, as previously reported (Oliveira et al., 2010), was developed.

The combination of active agent-loaded MPs with in situ gelling system was tried by a few groups with various particle-embedded hydrogels (Lagarce et al., 2005; Defail et al., 2006). In these studies, it was noticeable that during the in vitro release studies the amount of active agent release was decreasing, having a higher impact during the first hours, whose burst effect was almost eliminated. The incorporation of the MPs into the GG hydrogels strongly influenced both the profile and the amount of BSA and Dex released. As expected, hydrogels of higher GG concentration lead to a slower and more sustained release, independently of the processing conditions of the microparticulate system due to the higher density of the hydrogel which reduces the active agent diffusion. Moreover, the initial burst effect and the previously observed differences with the processing conditions, more notoriously for the $1.25 \%$ GG hydrogels, were attenuated. Active agents entrapped inside MPs have to diffuse through two barriers (particulate system and hydrogel) to be released into the surrounding media, which can reduce the burst effect and prolong the delivery (Chen et al., 2004; Sivakumaran et al., 2011). Thus, along with the time, the amount of released active agent will be mainly dependent on the degradation of the hydrogel. After 21 days of incubation, the GG hydrogels presented some signs of degradation after immersed in PBS for 3 weeks, which can be explained by hydrolytic reactions (Coutinho et al., 2010). However, a strong integration of the microparticulate system within the GG matrix was observed, even after the release studies, which shows the superior ability of these systems to maintain the particles within the defect site.

In summary, this work confirmed the capacity of PHBV microparticulate systems to deliver both hydrophilic and hydrophobic active agents, and determined how the organic phase composition can be defined to tailor their incorporation and respective release profile. Moreover, the embedding of these systems in injectable hydrogels confirmed that the proposed system provides a localized delivery avoiding uncontrolled migration, along with a sustained release of the active agents.

\section{Conclusion}

The main goal of this work was to propose a biphasic injectable DDS based on the combination of microparticulate systems and an injectable hydrogel for the localized delivery and longterm retention of MPs carrying hydrophilic and hydrophobic model active agents relevant for RM purposes, thus guaranteeing a prolonged release. In this sense, injectable GG hydrogels entrapping Dex- or BSA-loaded PHBV MPs were developed. Such platform was characterized by a prolonged release, which profile can be tailored according to the MPs and hydrogel features. The proposed system also offers the advantage of a high spatial control over the defect filling by a fast gelation at the injection site. Thus, the combination of an injectable GG hydrogel with Dex- or BSA-loaded PHBV MPs represents a promising strategy to deliver multiple active agents with distinct kinetics, which is likely required to drive tissue development to regeneration.

\section{Acknowledgments}

The authors would like to acknowledge the Project RL1 ABMR - NORTE-01-0124-FEDER-000016 co-financed by North Portugal Regional Operational Programme (ON.2 - O Novo Norte), under the National Strategic Reference Framework (NSRF), through the European Regional Development Fund (ERDF). This work was partially supported by European Research Council grant agreement ERC-2012-ADG 20120216-321266 for project ComplexiTE.

\section{References}

Abraham, G., Gallardo, A., San Román, J., Fernández-Mayoralas, A., Zurita, M., Vaquero, J., 2003. Polymeric matrices based on graft copolymers of PCL onto acrylic backbones for releasing antitumoral drugs. J. Biomed. Mater. Res. A 64 638-647.

Amass, W., Amass, A., Tighe, B., 1998. A review of biodegradable polymers: uses, current developments in the synthesis and characterization of biodegradable polyesters, blends of biodegradable polymers and recent advances in biodegradation studies. Polym. Int. 47, 89-144.

Balmayor, E., Tuzlakoglu, K., Azevedo, H., Reis, R., 2009. Preparation and characterization of starch-poly- $\varepsilon$-caprolactone microparticles incorporating bioactive agents for drug delivery and tissue engineering applications. Acta Biomater. 5, 1035-1045.

Bazzo, G. Lemos-Senna, E., Gonçalves, M., Pires, A., 2008. Effect of preparation conditions on morphology, drug content and release profiles of poly (hydroxybutyrate) microparticles containing piroxicam. J. Braz. Chem. Soc. 19, 914-921.

Bidone, J., Meloa, A.P.P., Bazzo, G.C., Carmignan, F., Soldi, M.S., Pires, A.T.N., Lemos-Senna, E., 2009. Preparation and characterization of ibuprofen-loaded microspheres consisting of poly(3-hydroxybutyrate) and methoxy poly 
(ethylene glycol)-b-poly(D,L-lactide) blends or poly(3-hydroxybutyrate) and gelatin composites for controlled drug release. Mater. Sci. Eng. C 29, 588-593.

Biondi, M., Ungaro, F., Quaglia, F., Netti, P.A., 2008. Controlled drug delivery in tissue engineering. Adv. Drug Delivery Rev. 60, 229-242.

Calandrelli, L., De Rosa, G., Errico, M.E., La Rotonda, M.I., Laurienzo, P., Malinconico, M., Oliva, A., Quaglia, F., 2002. Novel graft PLLA-based copolymers: potential of their application to particle technology. J. Biomed. Mater. Res. 62 244-253.

Champa, A., Bhat, A., 2010. Mesenchymal stem cell function on hybrid organic/ inorganic microparticles in vitro. J. Tissue Eng. Regen. Med. 4, 340-348.

Chan, B.P., So, K.F., 2005. Photochemical crosslinking improves the physicochemical properties of collagen scaffolds. J. Biomed. Mater. Res. A 75, 689-701.

Chen, B.H., Lee, D.J., 2001. Slow release of drug through deformed coating film: effects of morphology and drug diffusivity in the coating film. J. Pharm. Sci. 90, 1478-1496.

Chen, P.C., Kohane, D.S., Park, Y.J., Bartlett, R.H., Langer, R., Yang, V.C., 2004. Injectable microparticle-gel system for prolonged and localized lidocaine release. II. In vivo anesthetic effects. J. Biomed. Mater. Res. A 70, 459-466.

Chen, W., Tong, Y.W., 2012. PHBV microspheres as neural tissue engineering scaffold support neuronal cell growth and axon-dendrite polarization. Acta Biomater. 8, $540-548$.

Ciçek, H., Tuncel, A., Tuncel, M., Pişkin, E., 1995. Degradation and drug release characteristics of monosize polyethylcyanoacrylate microspheres. J. Biomater. Sci. Polym. Ed. 6, 845-856.

Correia, C.R., Reis, R.L., Mano, J.F., 2013. Multilayered hierarchical capsules providing cell adhesion sites. Biomacromolecules 14, 743-751.

Costa, P., Sousa Lobo, J.M., 2001. Modelling and comparison of dissolution profiles. Eur. J. Pharm. Sci. 13, 123-133.

Coutinho, D.F., Sant, S.V., Shin, H., Oliveira, J.T., Gomes, M.E., Neves, N.M., Khademhosseini, A., Reis, R.L., 2010. Modified gellan gum hydrogels with tunable physical and mechanical properties. Biomaterials 31, 7494-7502.

da Silva, L.P., Cerqueira, M.T., Sousa, R.A., Reis, R.L., Correlo, V.M., Marques, A.P., 2014 Engineering cell-adhesive gellan gum spongy-like hydrogels for regenerative medicine purposes. Acta Biomater. 10, 4787-4797.

Defail, A.J., Edington, H.D., Matthews, S., Lee, W.C., Marra, K.G., 2006. Controlled release of bioactive doxorubicin from microspheres embedded within gelatin scaffolds. J. Biomed. Mater. Res. A 79, 954-962.

Dhule, S.S., Penfornis, P., Frazier, T., Walker, R., Feldman, J., Tan, G., He, J., Alb, A. John, V., Pochampally, R., 2012. Curcumin-loaded $\gamma$-cyclodextrin liposomal nanoparticles as delivery vehicles for osteosarcoma. Nanomedicine 8 , 440-451.

Donbrow, M., Samuelov, Y., 1980. Zero order drug delivery from double-layered porous films: release rate profiles from ethyl cellulose, hydroxypropyl cellulose and polyethylene glycol mixtures. J. Pharm. Pharmacol. 32, 463-470.

Embleton, J.K., Tighe, B.J., 1993. Polymers for biodegradable medical devices. X Microencapsulation studies: control of poly-hydroxybutyrate-hydroxyvalerate microcapsules porosity via polycaprolactone blending. J. Microencapsul. 10 341-352.

Fitzgerald, P., Hadgraft, J., Wilson, C.G., 1987. A gamma scintigraphic evaluation of the precorneal residence of liposomal formulations in the rabbit. J. Pharm. Pharmacol. 39, 487-490.

Francis, L., Decheng, M., Knowles, J., Keshavarz, T., Boccaccini, A.R., Roy, I., 2011. Controlled delivery of gentamicin using poly(3-hydroxybutyrate) microspheres. Int. J. Mol. Sci. 12, 4294-4314.

Fulzele, S.V., Satturwar, P.M., Kasliwal, R.H., Dorle, A.K., 2004. Preparation and evaluation of microcapsules using polymerized rosin as a novel wall forming material. J. Microencapsul. 21, 83-89.

Gibaldi, M., Feldman, S., 1967. Establishment of sink conditions in dissolution rate determinations. Theoretical considerations and application to nondisintegrating dosage forms. J. Pharm. Sci. 56, 1238-1242.

Giunchedi, P., Conti, B., Maggi, L., Conte, U., 1994. Cellulose acetate butyrate and polycaprolactone for ketoprofen spray-dried microsphere preparation. J. Microencapsul. 11, 381-393.

Griffith, L.G., 2000. Polymeric biomaterials. Acta Mater. 48, 263-277.

Higuchi, T., 1961. Rate of release of medicaments from ointment bases containing drugs in suspension. J. Pharm. Sci. 50, 874-875.

Higuchi, T., 1963. Mechanism of sustained-action medication. Theoretical analysis of rate of release of solid drugs dispersed in solid matrices. J. Pharm. Sci. 52, $1145-1149$.

Igartua, M., Hernández, R.M., Esquisabel, A., Gascon, A.R., Calvo, M.B., Pedraz, J.L. 1997. Influence of formulation variables on the in-vitro release of albumin from biodegradable microparticulate systems. J. Microencapsul. 14, 349-356.

Iwanaga, S., Saito, N., Sanae, H., Nakamura, M., 2013. Facile fabrication of uniform size-controlled microparticles and potentiality for tandem drug delivery system of micro/nanoparticles. Colloids Surf. B 109, 301-306.

Jin, R., 2012. In-situ forming biomimetic hydrogels for tissue regeneration. In: Lin, C. (Ed.), Biomedicine, vol. 2. In Tech, Croatia, pp. 35-58.

Kabilan, S., Ayyasamy, M., Jayavel, S., Paramasamy, G., 2012. Pseudomonas sp. as a source of medium chain length polyhydroxyalkanoates for controlled drug delivery: perspective. Int. J. Micr. Res. 2012, 1-10.

Kassab, A.C., Xu, K., Denkbaş, E.B., Dou, Y., Zhao, S., Pişkin, E., 1997. Rifampicin carrying polyhydroxybutyrate microspheres as a potential chemoembolization agent. J. Biomater. Sci. Polym. Ed. 8, 947-961.

Korsmeyer, R.W., Gurnya, R., Doelkera, E., Buria, P., Peppas, N.A., 1983. Mechanism of solute release from porous hydrophilic polymers. Int. J. Pharm. 15, 25-35.
Korsmeyer, R.W., Peppas, N.A., 1981. Macromolecular and modeling aspects of swelling-controlled systems. In: Roseman, T., Mansdorf, S. (Eds.), Controlled Release Delivery Systems. Marcel Dekker, New York, pp. 77-90.

Kost, J., Langer, R., 2001. Responsive polymeric delivery systems. Adv. Drug Delivery Rev. 46, 125-148.

Kozielski, K.L., Tzeng, S.Y., Green, J.J., 2013. Bioengineered nanoparticles for siRNA delivery. Wiley Interdiscip. Rev. Nanomed. Nanobiotechnol. 5, 449-468.

Lagarce, F., Faisant, N., Desfontis, J.C., Marescaux, L., Gautier, F., Richard, J., Menei, P., Benoit, J.P., 2005. Baclofen-loaded microspheres in gel suspensions for intrathecal drug delivery: in vitro and in vivo evaluation. Eur. J. Pharm. Biopharm. 61, 171-180.

Lemperle, G., Morhenn, V.B., Pestonjamasp, V., Gallo, R.L., 2004. Migration studies and histology of injectable microspheres of different sizes in mice. Plast. Reconstr. Surg. 113, 1380-1390.

Liang, C.Z., Li, H., Tao, Y.Q., Peng, L.H., Gao, J.Q., Wu, J.J., Li, F.C., Hua, J.M., Chen, Q.X., 2013. Dual release of dexamethasone and transforming growth factor $\beta 3$ from polymeric microspheres for the stem cell matrix accumulation in a rat disc degeneration model. Acta Biomater. 9, 9423-9433.

Lionzo, M.I., Ré, M.I., Guterres, S.S., Pohlmann, A.R., 2007. Microparticles prepared with poly(hydroxybutyrate-co-hydroxyvalerate) and poly(epsiloncaprolactone) blends to control the release of a drug model. J. Microencapsul. 24, 175-186.

Liu, J., Xiao, Y., Allen, C., 2004. Polymer-drug compatibility: a guide to the development of delivery systems for the anticancer agent, ellipticine. J. Pharm. Sci. 93, 132-143.

Mi, F.L., Lin, Y.M., Wu, Y.B., Shyu, S.S., Tsai, Y.H., 2002. Chitin/PLGA blend microspheres as a biodegradable drug-delivery system: phase-separation, degradation and release behavior. Biomaterials 23, 3257-3267.

Monteiro, N., Martins, A., Ribeiro, D., Faria, S., Fonseca, N.A., Moreira, J.N., Reis, R.L., Neves, N.M., 2013. On the use of dexamethasone-loaded liposomes to induce the osteogenic differentiation of human mesenchymal stem cells. J. Tissue Eng. Regen. Med. doi:http://dx.doi.org/10.1002/term.1817 (in press).

Mukherjee, B., Santra, K., Pattnaik, G., Ghosh, S., 2008. Preparation, characterization and in-vitro evaluation of sustained release protein-loaded nanoparticles based on biodegradable polymers. Int. J. Nanomed. 3, 487-496.

Ogawa, Y., Yamamoto, M., Takada, S., Okada, H., Shimamoto, T., 1988. Controlledrelease of leuprolide acetate from polylactic acid or copoly(lactic/glycolic) acid microcapsules: influence of molecular weight and copolymer ratio of polymer. Chem. Pharm. Bull. 36, 1502-1507.

Oliveira, J.M., Sousa, R.A., Malafaya, P.B., Silva, S.S., Kotobuki, N., Hirose, M., Ohgushi, H., Mano, J.F., Reis, R.L., 2011. In vivo study of dendronlike nanoparticles for stem cells tune-up: from nano to tissues. Nanomedicine 7, 914-924.

Oliveira, J.T., Santos, T.C., Martins, L., Picciochi, R., Marques, A.P., Castro, A.G., Neves, N.M., Mano, J.F., Reis, R.L., 2010. Gellan gum injectable hydrogels for cartilage tissue engineering applications: in vitro studies and preliminary in vivo evaluation. Tissue Eng. Part A 16, 343-353.

Park, J., Ye, M., Park, K., 2005. Biodegradable polymers for microencapsulation of drugs. Molecules 10, 146-161.

Pinto Reis, C., Neufeld, R.J., Ribeiro, A.J., Veiga, F., 2006. Nanoencapsulation I. Methods for preparation of drug-loaded polymeric nanoparticles. Nanomedicine 2, 8-21.

Poletto, F.S., Fiel, L.A., Donida, B., Ré, M.I., Guterres, S.S., Pohlmann, A.R., 2008 Controlling the size of poly(hydroxybutyrate-co-hydroxyvalerate) nanoparticles prepared by emulsification-diffusion technique using ethanol as a surface agent. Colloids Surf. A 324, 105-112.

Rodríguez-Contreras, A., Canal, C., Calafell-Monfort, M., Ginebra, M.P., Julio-Moran, G., Marqués-Calvo, M.S., 2013. Methods for the preparation of doxycycline-loaded phb micro- and nano-spheres. Eur. Polym. J. 49, 3501-3511.

Sah, H.K., Toddywala, R., Chien, Y.W., 1994. The influence of biodegradable microcapsule formulations on the controlled release of a protein. J. Controlled Release 30, 201-211.

Sahoo, S.K., Panyam, J., Prabha, S., Labhasetwar, V., 2002. Residual polyvinyl alcohol associated with poly(D,L-lactide-co-glycolide) nanoparticles affects their physical properties and cellular uptake. J. Controlled Release 82, 105-114.

Shishatskaya, E.I., Voinova, O.N., Goreva, A.V., Mogilnaya, O.A., Volova, T.G., 2008. Biocompatibility of polyhydroxybutyrate microspheres: in vitro and in vivo evaluation. J. Mater. Sci. Mater. Med. 19, 2493-2502.

Sivakumaran, D., Maitland, D., Hoare, T., 2011. Injectable microgel-hydrogel composites for prolonged small-molecule drug delivery. Biomacromolecules $12,4112-4120$.

Soppimath, K.S., Aminabhavi, T.M., Kulkarni, A.R., Rudzinski, W.E., 2001. Biodegradable polymeric nanoparticles as drug delivery devices. J. Controlled Release 70, 1-20.

Tunón, A., Gråsjö, J., Alderborn, G., 2003. Effect of intragranular porosity on compression behaviour of and drug release from reservoir pellets. Eur. J. Pharm. Sci. 19, 333-344.

Wagner, J.G., 1969. Interpretation of percent dissolved-time plots derived from in vitro testing of conventional tablets and capsules. J. Pharm. Sci. 58, 1253-1257.

Wen, Y., Gallego, M.R., Nielsen, L.F., Jorgensen, L., Møller, E.H., Nielsen, H.M., 2013. Design and characterization of core-shell mPEG-PLGA composite microparticles for development of cell-scaffold constructs. Eur. J. Pharm. Biopharm. 85, 87-98.

Xu, L., Xu, X., Chen, H., Li, X., 2013. Ocular biocompatibility and tolerance study of biodegradable polymeric micelles in the rabbit eye. Colloids Surf. B 112, 30-34.

Yang, Y.Y., Chung, T.S., Ng, N.P., 2001. Morphology, drug distribution, and in vitro release profiles of biodegradable polymeric microspheres containing protein 
fabricated by double-emulsion solvent extraction/evaporation method. Biomaterials 22, 231-241.

Yeo, Y., Baek, N., Park, K., 2001. Microencapsulation methods for delivery of protein drugs. Biotechnol. Bioprocess Eng. 6, 213-230.

Zhang, Y., Chu, C.C., 2002. In vitro release behavior of insulin from biodegradable hybrid hydrogel networks of polysaccharide and synthetic biodegradable polyester. J. Biomater. Appl. 16, 305-325.
Zhu, X.H., Wang, C.H., Tong, Y.W., 2007. Growing tissue-like constructs with Hep3B/ HepG2 liver cells on PHBV microspheres of different sizes. J. Biomed. Mater. Res. B Appl. Biomater. 82, 7-16.

Zonari, A., Cerqueira, M.T., Novikoff, S., Goes, A.M., Marques, A.P., Correlo, V.M., Reis, R.L., 2014. Poly(hydroxybutyrate-co-hydroxyvalerate) bilayer skin tissue engineering constructs with improved epidermal rearrangement. Macromol. Biosci. 14, 977-990. 